\title{
Control and Operation of a Hybrid Actuator for Maglev Applications
}

Research Article

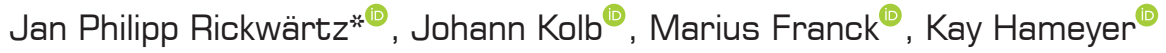 \\ RWTH Aachen University, Faculty of Electrical Engineering and Information Technology, Institute of Electrical Machines and Chair in Electromagnetic \\ Energy Conversion, Schinkelstraße 4, 52062 Aachen, Germany
}

Received: June 15, 2021; Accepted: August 18, 2021

\begin{abstract}
The hybrid actuator presented in this article is meant to enable stationary and slow dynamic levitation in Maglev applications. The term 'hybrid' refers to the design of the actuator, which is a combination of permanent magnets (PM) and electromagnets. This paper presents an analytically computable control algorithm for the said hybrid actuator. The theory of magnetic circuits is summarized shortly and used to derive a cascaded control loop consisting of an inner current controller and an outer air gap controller. Since the uncontrolled hybrid actuator is inherently unstable, the system has to be stabilized. By introducing a PID-controller into the air gap control loop, the unstable behaviour of the uncontrolled system is changed into the system behaviour of a damped harmonic oscillator. The advantage of this approach is that the computed controller parameters of the PID-controller can easily be adjusted, so the system behaviour of damping and eigenfrequency can be selected within a certain range. For the execution of the control algorithm, a microcontroller (MCU) is used and for precise air gap measurement, an eddy current sensor is installed. Finally, the behaviour of the current- and air gap controller is discussed for different measurement results and the adjustable system behaviour of the damped harmonic oscillator is presented.
\end{abstract}

Keywords: Maglev • electromagnetic levitation • controlled permanent magnet • hybrid actuator

\section{Introduction}

The benefits of magnetically levitated actuator systems are discussed in various publications presenting numerous ideas of applications. Some of these proposals have already been implemented commercially, others are only implemented as prototypes or are still part of considerations and studies. In any case, the potential of magnetic levitation is high, and this technology will likely be able to solve certain challenges of the future. Probably the bestknown application of this technology can be seen in magnetically levitated highspeed trains such as the German 'Transrapid' (Bohn and Steinmetz, 1984), whose levitation technology is based on electromagnetic suspension (EMS) or the Japanese 'JR-Maglev' railway system (He et al., 1994), whose technology is based on electrodynamic suspension (EDS). Apart from these popular fields of application, magnetically levitated actuator systems are also used in active bearings (Henzel and Mazurek, 2011; Zhang et al., 2010), enabling operation at very high speeds. In manufacturing environments where e.g. a high level of cleanliness is required, such as in clean rooms, magnetically levitated conveyors can be a solution to offer abrasion-free operation (Kim et al., 2013; Shin et al., 2016). In both fields of operation, applications benefit from controlled vibration reduction due to the absence of mechanical coupling, if the actuator system is properly controlled (Lin and Lin, 2000). The realisation of EMS, as it is based on controlling magnetic field strength in a magnetic circuit, can be done by using electromagnets only. In this case, field strength and therefore the complete actuating force is controlled by the current in the actuator's coils (Cervera et al., 2019). The alternative is to add permanent magnets (PM) to the electromagnets, which impose, with regard to a certain operational point in the magnetic circuit, a static force. Therefore, electromagnets can be used for compensating only dynamic loads while PM hold the static load. Since the uncontrolled PM-actuator is inherently unstable, the challenge is to design the actuator for its intended operating range and equip it with a stabilising 
control algorithm offering as high dynamics as possible. If permanent magnets are used, then at the intended operating point no DC current component is necessary to form a force that keeps the actuator in suspension. This case is referred to as zero current respectively zero power operation (Kim et al., 2001; Kim et al., 2010). Current is only needed to dynamically stabilise the system if it leaves its operation point due to disturbances. If a variable flux path is used, this principle is also adaptable to systems with varying operational points (Zhao et al., 2020).

In an initial publication (Rickwartz et al., 2020), the focus was on implementing a PM assisted levitation actuator to magnetically levitate a Maglev train model on a short track at standstill and very low speed while the linear motor is not in operation or just starts its operation. In this paper, a very general approach is provided to control a PM assisted levitation actuator (hybrid actuator) as shown in Figure 1, whose principle can be used in numerous applications. The hybrid actuator consists of a U-shaped iron yoke with a cross-section of $20 \times 20 \mathrm{~mm}$, two high-energy NdFeBmagnets of $20 \times 20 \times 1 \mathrm{~mm}$ size, assembled on each leg of the yoke and two coils with 70 windings each, which are pulled over the legs of the yoke. The winding diameter is $1 \mathrm{~mm}$ without insulation. Together with the iron yoke of the single actuator test bench, which the hybrid actuator is mounted on, the magnetic circuit with variable air gap is closed and can be described by basic theory in Section 2 . The derivation results in an analytical equation to describe the total magnetic force that the hybrid actuator can apply. This simple, but widely applicable approach can then be adapted to other, more specific applications (Cho et al., 2012). In Section 3, the controller design is presented and divided into an electrical subsystem, consisting of a current-controlled inductance-resistance element (LR-element) and a mechanical subsystem starting with the dynamic equation of motion. While the static load, so the weight force of the levitated object, is assumed to be compensated by the force of the magnets, dynamic changes caused by any kind of disturbance result in an acceleration of the actuator. These dynamic changes have to be controlled by applying dynamic forces through changing magnetic field strength in the magnetic circuit by applying currents in the coils. Therefore, the unstable transfer function of the uncontrolled, dynamic system is extended by the transfer function of a PID-controller. The resulting transfer function can be compared to the transfer function of a damped harmonic oscillator with adjustable spring- and damping constant by tuning PID-controller parameters. Section 4 explains the test bench setup and discusses the results of the measurements to validate the theoretical studies.

\section{Basic Theory}

This section repeats and collects the basic theory of electromagnetic fields generating pulling forces which realise the principle of magnetic levitation. In Figure 1, the topology of the magnetic circuit of the hybrid actuator is shown. The generation of magnetic flux is divided into two components: The first component is electromagnetic, where copper coils of the winding number $N$ are energised with current $I$ of variable amplitude and frequency, leading to controllable magnetic field strength $H$. This relation is described by Ampère's law

$$
\oint \vec{H} \cdot \overrightarrow{d s}=N \cdot I=\Theta .
$$

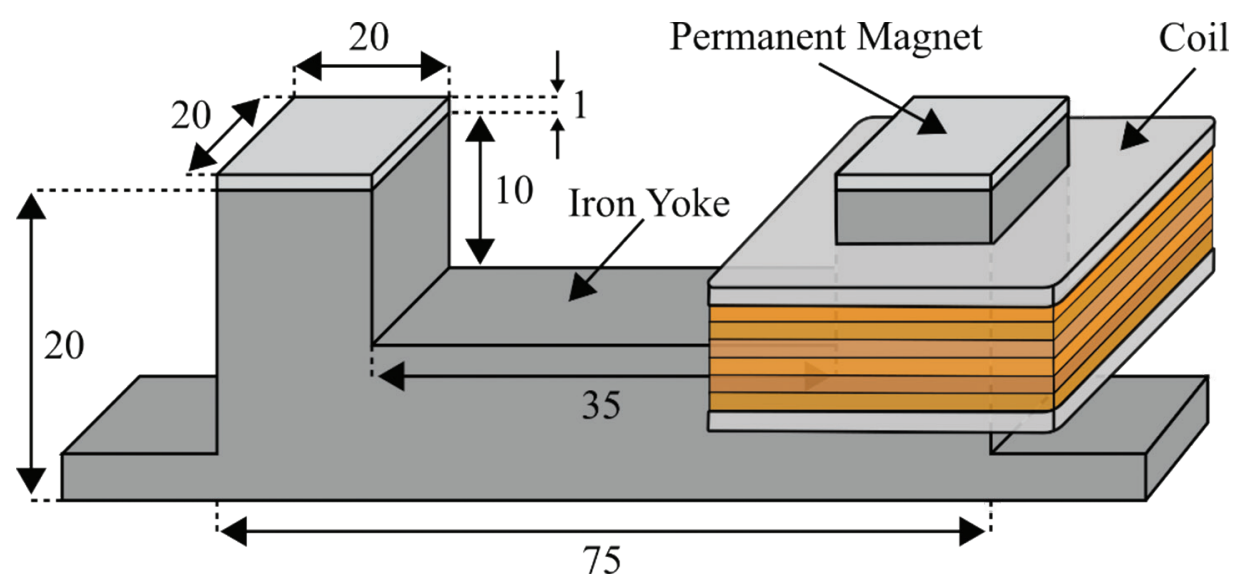

Fig. 1. Schematic of the hybrid-actuator (dimensions in $\mathrm{mm}$ ). 
The generation of variable magnetomotive force $\Theta$ is supplemented by the second flux component, the PM. The flux density of the magnets $B_{P M}$ is therefore a combination of the characteristic remanence $B_{R}$, the relative permeability $\mu_{r, P M}$ of the used NdFeB-magnets and the external magnetic field strength in the magnets $H_{P M}$. With the magnetic field constant $\mu_{0}$, the external flux density of the magnets can be described as

$$
B_{P M}=B_{R}+\mu_{0} \cdot \mu_{r, P M} \cdot H_{P M}
$$

Due to the plain geometry of the hybrid actuator, the path integral of the magnetic field strength can be approximated by areas of constant magnetic field strength and the respective mean path length of the iron yoke $I_{F E}$, two air gaps $\delta_{L}$ and two magnets $h_{P M}$

$$
N \cdot I=H_{F e} \cdot l_{F e}+2 \cdot H_{P M} \cdot h_{P M}+2 \cdot H_{a i r} \cdot \delta_{L}
$$

For yoke and air gap, the relationship between field strength and flux density can be deduced from the general material equation $B=\mu_{0} \cdot \mu_{r} \cdot H$ and for the PM from Eq. (2). By inserting these equations into Eq. (3), the relationship between current and magnetic flux density $B$ follows

$$
N \cdot I=\frac{B_{F e}}{\mu_{0} \cdot \mu_{r, F e}} \cdot l_{F e}+2 \cdot \frac{B_{P M}-B_{R}}{\mu_{0} \cdot \mu_{r, P M}} \cdot h_{P M}+2 \cdot \frac{B_{a i r}}{\mu_{0}} \cdot \delta_{L} .
$$

If the relative permeability of the iron yoke $\mu_{r, F e}$ is assumed to be very high compared to the relative permeability of the surrounding air $\left(\mu_{r, a i r} \approx 1\right)$ and the air gap length $\delta_{L}$ is small in relation to the cross-section $A$ of the flux guiding iron, fringing flux can be neglected for all following considerations. If the cross-section $A$ of the magnetic circuit is constant, the magnetic flux densities of all areas are equal $\left(B_{F e}=B_{P M}=B_{a i r}\right)$ and thus Eq. (4) can be simplified to

$$
N \cdot I=2 \cdot \frac{B_{a i r}-B_{R}}{\mu_{0} \cdot \mu_{r, P M}} \cdot h_{P M}+2 \cdot \frac{B_{a i r}}{\mu_{0}} \cdot \delta_{L} .
$$

This leads to an expression of air gap flux density $B_{\text {air }}$ dependent on current $I$ and air gap $\delta_{L}$

$$
B_{\text {air }}=\frac{N \cdot I \cdot \mu_{0} \cdot \mu_{r, P M}}{2 \cdot\left(h_{P M}+\delta_{L} \cdot \mu_{r, P M}\right)}+\frac{B_{R} \cdot h_{P M}}{h_{P M}+\delta_{L} \cdot \mu_{r, P M}} .
$$

Given that the provided assumptions and simplifications are still valid, the total force of the hybrid actuator $F_{\text {mag }}$ is introduced by Maxwell's pulling force formula and can be derived from the differential of energy for a certain air gap change.

$$
F_{M a g}=\frac{\mathrm{dW}}{\mathrm{d} \delta_{L}}=2 \cdot \frac{B_{a i r}^{2} \cdot A_{P M}}{2 \cdot \mu_{0}}=\frac{B_{a i r}^{2} \cdot A_{P M}}{\mu_{0}} .
$$

Inserting Eq. (6) into Eq. (7) leads to the total magnetic force $F_{\text {mag }}$ of the hybrid actuator depending on the current $I$ energising the coils, the remanent flux density $B_{R}$ of the PMs and the air gap $\delta_{L}$

$$
F_{M a g}=\frac{A_{P M}}{\mu_{0}} \cdot\left(\frac{N \cdot I \cdot \mu_{0} \cdot \mu_{r, P M}+2 \cdot B_{R} \cdot h_{P M}}{2 \cdot\left(h_{P M}+\delta_{L} \cdot \mu_{r, P M}\right)}\right)^{2} .
$$

\section{Controller Design}

The topology of the controller design is shown in Figure 2. The cascaded control loop consists of an inner proportional-integral (PI) current controller, calculating the duty cycle, which is applied to the $\mathrm{H}$-bridge energising the coils of the hybrid actuator, which are modelled as LR-element. The difference between current measurement 


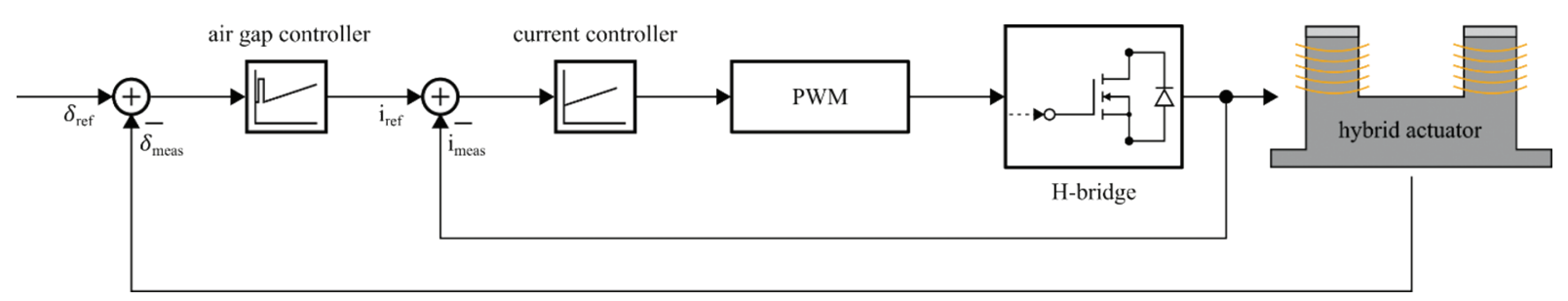

Fig. 2. Block diagram of the cascaded control loop.

and current reference coming from the outer PID air gap controller is fed into the current controller as the input signal. The input of the air gap controller is given by the difference between the measured air gap signal and air gap reference. In this section, controller parameters for current and air gap controllers are derived by mapping the physical properties of the hybrid actuator to transfer functions.

\subsection{Electrical subsystem}

The voltage equation of the coil, which is modelled as a combination of resistance $R$ and inductance $L$, can be described by

$$
u(t)=R i(t)+\frac{d}{d t} \psi\left(i, \delta_{L}\right) .
$$

Inserting the inductance, which is dependent on the air gap position and the time-dependent current for the flux linkage $\left(\psi\left(i, \delta_{L}\right)=L\left(\delta_{L}\right) i\right)$, the voltage equation can be rewritten as

$$
u(t)=R i(t)+L\left(\delta_{L}\right) \frac{d i(t)}{d t}-\frac{d L\left(\delta_{L}\right)}{d \delta_{L}(t)} \dot{\delta}_{L}(t) i(t) .
$$

With the assumption of small air gap changes within one control cycle, the induced voltage caused by air gap position changes can be neglected and after performing the Laplace transform, the transfer function is outlined as

$$
G_{C}=\frac{I(s)}{U(s)}=\frac{\frac{1}{R}}{\frac{L}{R} s+1}=\frac{\frac{1}{R}}{T_{l} s+1} .
$$

For the current controller, a PI control structure is applied. To evaluate the optimal control parameters, the magnitude optimum criterion is utilised (Papadopoulos, 2015). According to the rules of the magnitude optimum, the larger time constant of the controlled system $T_{\text {, }}$ has to be compensated with the controller time constant $T_{N, e l}$

$$
T_{N, e l}=T_{l}=\frac{L}{R} .
$$

Besides a large time constant $T_{\mu}$, a smaller time constant $T_{s}$ is needed to apply the rules of magnitude optimum. The physical background of $T_{s}$ is the dead time of power electronics and additional delays in the control loop, such as signal propagation time and processing time. The dead time is caused by the current measurement, which is triggered twice in a pulse-width modulation (PWM) period at the beginning and in the middle of each period for centre aligned PWM. Therefore, the small-time constant can be approximated to a value between half of a PWM period, which would only take the dead time into account without additional delay and a complete PWM period. Here, a small-time constant is approximated to be $T_{P W M}=1 / f_{P W M}=T_{S}$ (Bierhoff and Fuchs, 2009). The complete transfer function of the open control loop of the electrical subsystem results in

$$
G_{o, e l}(s)=K_{P, e l} \cdot \frac{T_{N, e l} s+1}{T_{N, e l} s} \cdot \frac{\frac{1}{R}}{T_{l} s+1} \cdot \frac{1}{T_{s} s+1} .
$$


According to the rules of the magnitude optimum, the controller gain $K_{P, e l}$ is calculated so that the absolute value of the reference transfer function is kept close to one for the largest possible frequency range, resulting in

$$
K_{P, e l}=\frac{T_{N, e l}}{2 K_{S} T_{E}}=\frac{L}{2 T_{s}},
$$

where $K_{S}$ is the static gain of the numerator of the open loop transfer function and $T_{E}$ the smaller time constant of the transfer function $G_{0, e l}$. Consequently, the resulting transfer function of the closed current control loop is given by

$$
G_{c, e l}(s)=\frac{G_{o, e l}}{1+G_{o, e l}}=\frac{1}{2 T_{s} s\left(1+T_{s} s\right)+1} .
$$

\subsection{Mechanical subsystem}

The mechanical equation results from the balance of forces, where $F_{\text {mag }}$ is the magnetic force derived in Eq. (8), $m g$ is the weight force, where $m$ is the mass and $g$ is the gravitational acceleration and $m \delta_{L}$ is the inertial force.

$$
0=F_{\text {Mag }}\left(I, \delta_{L}\right)-m g+m \ddot{\delta}_{L}
$$

Because $F_{M a g}\left(\delta_{L}, I\right)$ is nonlinear, the differential equation has to be linearised to evaluate the controller parameters. The resulting equation is split into a static and a dynamic equation. The static equation is defined as

$$
F_{\text {Mag }}\left(I_{0}, \delta_{L, 0}\right)=m \cdot g
$$

As described in Sections 1 and 2, the hybrid actuator is designed so that the weight force of the actuator and additional load can be compensated by the force, generated by the PM. Therefore, the static current can be set to $I_{0}=0 A$. Dynamic changes of the air gap, load changes and disturbances have to be controlled by monitoring magnetic field strength in the magnetic circuit, which results in controlling currents in the coils as explained in Section 1. Without the static part, the mechanical Eq. (16) can be simplified to the dynamic balance of forces.

$$
0=F_{\text {Mag }}\left(I, \delta_{L}\right)+m \ddot{\delta}_{L}
$$

This differential equation can then be linearized around an operational point $\left(I_{0}, \delta_{L, 0}\right)$

$$
0=k_{I, M a g} \Delta I+k_{\delta, M a g} \Delta \delta_{L}+m \Delta \ddot{\delta}_{L}
$$

where the current and air gap difference are defined as $\Delta I=I-I_{0}$ and $\Delta \delta_{L}=\delta_{L}-\delta_{L, 0}$. The static air gap is defined as the design air gap $\delta_{L, 0}$. The constant factors $k_{I, M a g}$ and $k_{\delta, M a g}$ result from the magnetic force linearisation in the operating point.

$$
\begin{aligned}
& k_{I, \text { Mag }}=\frac{A_{P M} \cdot N \cdot \mu_{r, P M} \cdot\left(I_{0} \cdot N \cdot \mu_{0} \cdot \mu_{r, P M}+2 \cdot B_{R} \cdot h_{P M}\right)}{2 \cdot\left(h_{P M}+\delta_{L, 0} \cdot \mu_{r, P M}\right)^{2}}, \\
& k_{\delta, M a g}=-\frac{A_{P M} \cdot \mu_{r, P M} \cdot\left(I_{0} \cdot N \cdot \mu_{0} \cdot \mu_{r, P M}+2 \cdot B_{R} \cdot h_{P M}\right)^{2}}{2 \cdot \mu_{0} \cdot\left(h_{P M}+\delta_{L, 0} \cdot \mu_{r, P M}\right)^{3}} .
\end{aligned}
$$

With the Laplace transform, the resulting transfer function of Eq. (19) is

$$
G_{A}(s)=\frac{-k_{I, M a g}}{k_{\delta, M a g}+m s^{2}} .
$$



From Eq. (22) follows that the mechanical system is unstable with poles at $s_{1 / 2}= \pm \sqrt{\frac{-k_{\delta, M a g}}{m}}$. To reach an
asymptotically stable system condition, all poles have to be in the left half of $s$-plane.

In contrast to the system behaviour of the hybrid actuator, a stable and physically achievable system can be depicted by the behaviour of a damped harmonic oscillator. That means a second-order mechanical differential equation describing the dynamics of a mass, which is mounted on a spring-damper-element with damping constant $d$ and spring constant $c$

$$
0=m \Delta \dot{\delta}_{L}+d \Delta \dot{\delta}_{L}+c \Delta \delta_{L}
$$

If Eq. (19) is equated with Eq. (23), the resulting differential Eq. (24) can be transformed by the Laplace transform into the corresponding transfer function (25) resulting in the transfer behaviour between the air gap and current dependent on $k_{I, M a g}, k_{\delta, M a g}$ and $c, d$

$$
\begin{aligned}
& \Delta I=\frac{d}{k_{I, M a g}} \Delta \dot{\delta}_{L}+\frac{c-k_{\delta, M a g}}{k_{I, M a g}} \Delta \delta_{L}, \\
& G_{\delta}(s)=\frac{\Delta I}{\Delta \delta_{L}}=\frac{c-k_{\delta, M a g}}{k_{I, M a g}}\left(\frac{d}{c-k_{\delta, M a g}} s+1\right) .
\end{aligned}
$$

The transfer function in Eq. (25) is equal to the transfer function of an ideal proportional-derivative (PD) controller $G_{P D}(s)$ given in Eq. (26)

$$
G_{P D}(s)=G_{\delta}(s)=K_{P D}\left(T_{V} s+1\right),
$$

where $K_{P D}$ and $T_{V}$ are the parameters of the PD controller and are determined to

$$
\begin{aligned}
& K_{P D}=\frac{c-k_{\delta, M a g}}{k_{I, M a g}}, \\
& T_{V}=\frac{d}{c-k_{\delta, M a g}} .
\end{aligned}
$$

If the required transfer behaviour between the air gap and current is described by the transfer function of the PD controller $G_{P D}(s)$ introduced in Eqs (26)-(28) and the mechanical transfer behaviour of the hybrid actuator is given by the dynamic balance of forces $G_{A}(s)$ in Eq. (22), the open-loop transfer function of the air gap control loop can be written as

$$
G_{o, P D}(s)=G_{P D}(s) G_{A}(s)=\frac{-k_{I, M a g} K_{P D}\left(T_{V} s+1\right)}{m s^{2}+k_{\delta, M a g}} .
$$

This is valid under the assumption that the control loop of the current controller can be approximated by a transfer behaviour of one for all relevant operating points. This is achieved by designing the current controller so that it is much faster than the air gap controller and stable for all relevant operating points. The closed-loop transfer function (30) of the air gap control loop has the desired transfer behaviour of a damped harmonic oscillator. The system behaviour is adjustable by parameterising $K_{P D}$ and $T_{V}$

$$
G_{c, P D}(s)=\frac{G_{o, P D}(s)}{1+G_{o, P D}(s)}=\frac{-k_{I, M a g} K_{P D}\left(T_{V} s+1\right)}{m s^{2}-k_{I, M a g} K_{P D} T_{V} s-k_{I, M a g} K_{P D}+k_{\delta, M a g}} .
$$

The stability of the system is analysed by calculating the poles of $G_{c, P D}(s)$ 


$$
s_{1,2}=\frac{k_{I, M a g} K_{P D} T_{V}}{2 m} \pm \sqrt{\left(\frac{k_{I, M a g} K_{P D} T_{V}}{2 m}\right)^{2}-\frac{k_{\delta, M a g}-k_{I, M a g} K_{P D}}{m}} .
$$

To achieve stable system behaviour, a double pole in the left half of the $s$-plane is intended. Therefore, the square root of Eq. (31) must be zero. By inserting $K_{P D}$ and $T_{V}$, a relation between $d$ and $c$ can be formulated

$$
d=-\sqrt{4 m\left(2 k_{\delta, M a g}-c\right)}
$$

where $\left(2 k_{\delta, M a g}-c\right)>0$ has to be fulfilled. According to this constraint, the value of $c$ has to be chosen and thus determines the location of the poles. Since the system behaviour corresponds to the behaviour of a damped harmonic oscillator, the characteristic parameters can be calculated. The natural eigenfrequency $f_{0}$ is given by

$$
f_{0}=\frac{\omega_{0}}{2 \pi}=\frac{1}{2 \pi} \sqrt{\frac{c}{m}}
$$

and the damped eigenfrequency $f_{d}$ is calculated to

$$
f_{d}=\frac{\omega_{d}}{2 \pi}=\frac{\omega_{0}}{2 \pi} \sqrt{1-\zeta^{2}}
$$

with the damping ratio $\zeta$ expressed as

$$
\zeta=\frac{d}{2 m \omega_{0}}
$$

If all poles of the transfer function in Eq. (30) are in the left half-plane and the closed transfer function is examined according to the final value theorem in Eq. (36), it can be found that for a unit step, with a reference signal $W(s)=\frac{1}{s}$, a stationary control deviation occurs. Thus, the remaining control deviation can be calculated with

$$
\lim _{t \rightarrow \infty} e(t)=\lim _{s \rightarrow 0} s^{2}(W(s)-Y(s))=\lim _{s \rightarrow 0} s^{2} W(s)\left(1-\frac{G_{0}(s)}{1+G_{0}(s)}\right),
$$

where $e$ is the measured error, $W(s)$ is the reference signal, $Y(s)$ is the system output and $G_{0}(s)$ is the open-loop transfer function.

Therefore, the PD controller behaviour is replaced by a PID controller, to eliminate the remaining control deviation between setpoint and actual value. An ideal PID controller has the following structure.

$$
G_{P I D}(s)=K_{P I D}\left(1+\frac{1}{T_{N} s}+T_{V} s\right)
$$

The characteristic of the PD part of the PID controller has to be the same as for the PD controller. Therefore, $K_{P I D}$ is set to

$$
K_{P I D}=K_{P D}=\frac{c-k_{\delta, M a g}}{k_{I, M a g}} .
$$

If Eq. (37) is inserted in Eq. (29) instead of $G_{P D}(s)$, the open-loop transfer function is given by

$$
G_{o, P I D}(s)=\frac{-k_{I, M a g} K_{P I D}\left(1+\frac{1}{T_{N} s}+T_{V} s\right)}{m s^{2}+k_{\delta, M a g}}
$$


with the resulting closed-loop transfer function.

$$
G_{c, P I D}(s)=\frac{G_{o, P I D}(s)}{1+G_{o, P I D}(s)}=\frac{-k_{I, M a g} K_{P I D}\left(T_{N} T_{V} s^{2}+T_{N} s+1\right)}{T_{N} m s^{3}+k_{I, M a g} K_{P I D} T_{N} T_{V} s^{2}+\left(k_{\delta, M a g}-k_{I, M a g} K_{P I D}\right) T_{N} s-k_{I, M a g} K_{P I D}} .
$$

To achieve stable system behaviour, the poles of the closed-loop transfer function (40) have to be determined. The selection of the reset time $T_{N}$ is then dependent on the trade-off between overshooting and duration until a control deviation should be eliminated. For the application in this paper, a well-damped system behaviour is reached by setting $T_{N}$ as a multiple of $T_{V}$.

\section{Measurements}

To study the behaviour of the current and air gap controller, a hybrid actuator test bench is designed. Different measurements are performed to validate the system behaviour, which was expounded in Section 3 .

\subsection{Test bench setup}

As already mentioned in Section 1 and illustrated in Figure 1, the hybrid actuator consists of an iron yoke with two legs and a copper coil of 70 windings pulled over each leg of the yoke. On top of each leg, a NdFeB-magnet is mounted. The magnetic circuit is closed by the iron yoke of the hybrid actuator test bench shown in Figure 3. An eddy current displacement sensor is assembled close to the hybrid actuator, measuring the air gap and an iron weight is placed on the test bench, which results in an effective mass of $5.3 \mathrm{~kg}$, if the lever is considered. The moving range of the actuator is limited between $0.5 \mathrm{~mm}$ and $1.5 \mathrm{~mm}$ during operation. An air gap of $1.5 \mathrm{~mm}$ is selected as a proper starting point, where a limited current still results in enough force to attract the actuator and $0.5 \mathrm{~mm}$ are selected to prevent the actuator from attracting itself completely to the iron yoke. With a calculated time constant $T_{V}$ of $10 \mathrm{~ms}$, the air gap controller reset time is selected according to Section 3.2 as a multiple of $T_{V}$ to $T_{N}=90 \mathrm{~ms}$.

The control algorithm is implemented on an ST Nucleo-F767ZI microcontroller (MCU) shown in Figure 4 (left). For air gap and current measurement, a custom-built shield board (Figure 4 centre) is used. The shield board is mounted on the MCU board equipped with a 16-channel 16-Bit dual simultaneous sampling Analog Devices AD7616 (ADC), power supply, PWM outputs and has furthermore measurement inputs for six $\mathrm{H}$-bridges. For the data acquisition, the SPI bus sends data from $A D C$ to $M C U$, which runs the control algorithm and forwards data to the measurement computer over Universal Serial Bus On-The-Go (USB OTG) interface. Figure 4 (right) shows the H-bridge, which consists of two half bridges (International Rectifier IRSM808-204MH), a LEM LAX 100-NP for current measurement, an air gap signal evaluation circuit and a power supply for the air gap amplifier. The sensor for measuring the air gap distance between actuator and iron yoke is an eddy current sensor of type Micro-Epsilon eddyNCDT 3005.

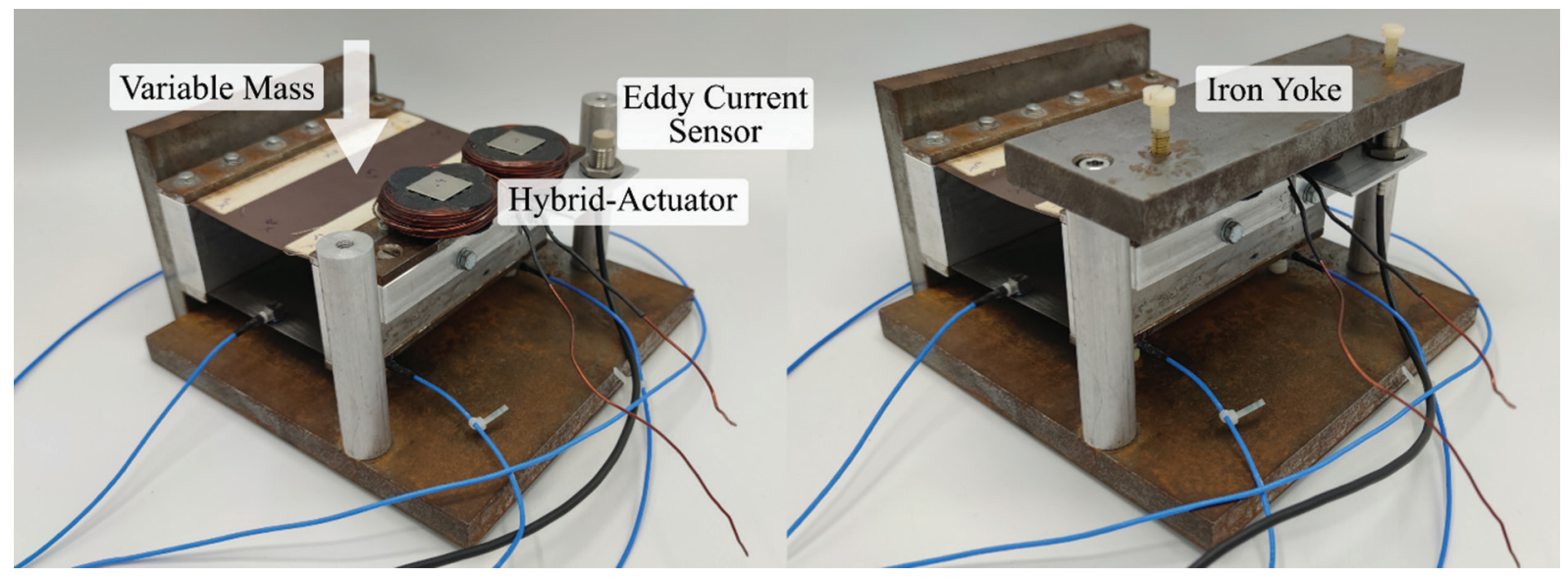

Fig. 3. Hybrid actuator test bench. 
Because the PM vary slightly in their remanence, the reference air gap is measured at zero current to evaluate the exact controller parameters. To identify the respective air gap value, a simple integral controller is placed into the cascaded control loop, as shown in Figure 5. The current measurement value is integrated and subtracted from the air gap setpoint value. As soon as the current reaches zero, the air gap value settles. In Figure 6, the air gap measurement value settles at $1.11 \mathrm{~mm}$.
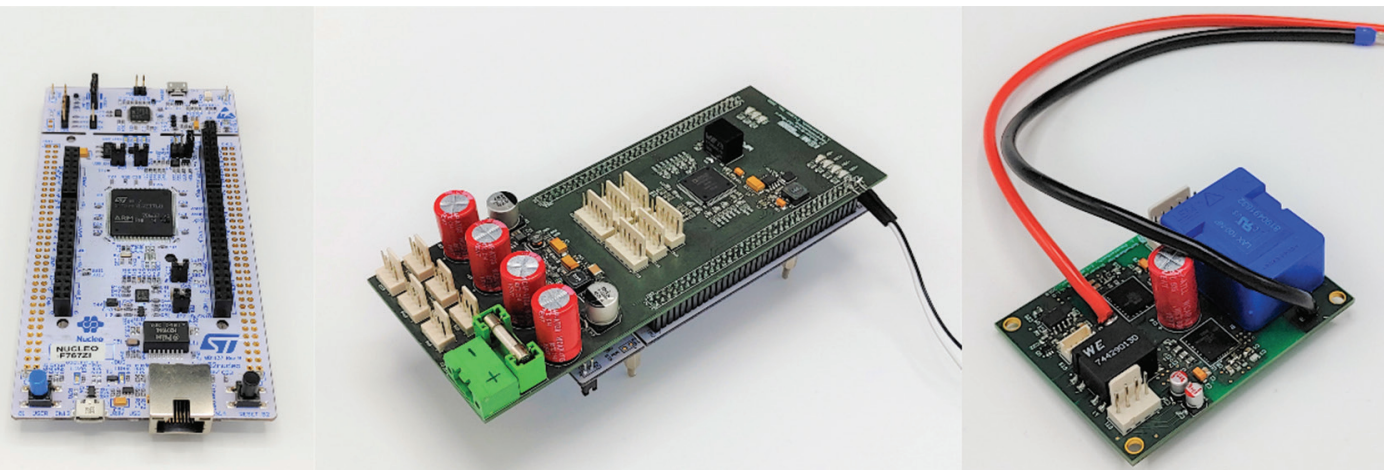

Fig. 4. $M C U$ (left), shield board (centre) and power electronics board (right)

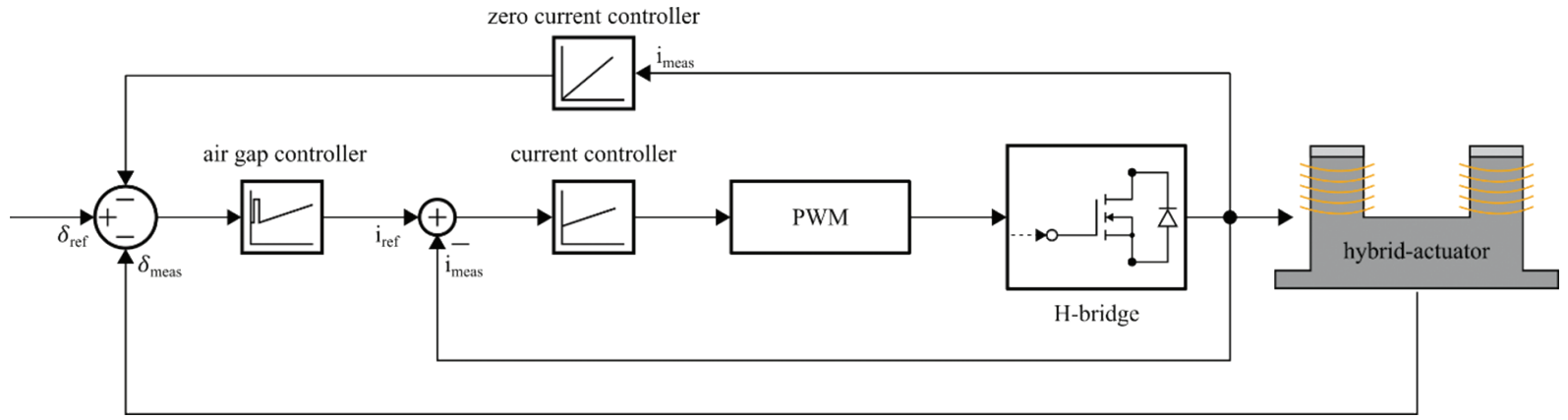

Fig. 5. Block diagram of the cascaded control loop and zero current controller.
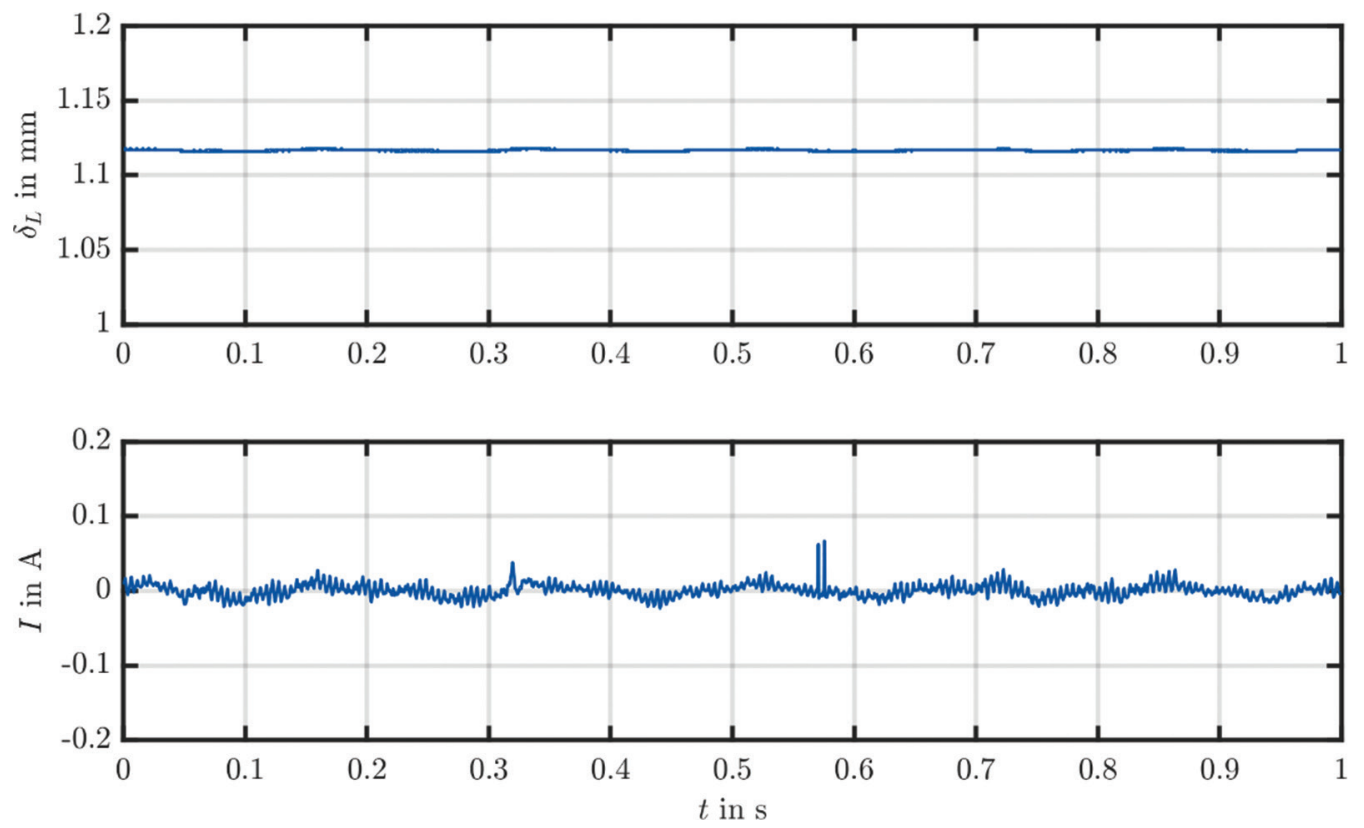

Fig. 6. Measurement to identify the air gap at zero current. 
With the knowledge of the system parameters given in Table 1, the controller parameters can be derived from the equations in Section 3. The controller parameters, belonging to the parameters in Table 1, are given in Table 2. As the controlled system behaves like a damped harmonic oscillator, as outlined in Section 3.2, the damped eigenfrequency can be calculated to $f_{D}=22.1 \mathrm{~Hz}$.

\subsection{Evaluation}

In this section, measurement results, obtained with the hybrid actuator test bench and the measurement electronics used, are discussed and compared to the theoretical system behaviour. First, the inner current control loop is analysed. The upper diagram of Figure 7 illustrates a set point current ramp with a slope of $4 \mathrm{~A} / \mathrm{ms}$ rising from $0 \mathrm{~A}$ to $2 \mathrm{~A}$ and the current measurement value measured with the ADC at carrier peak of the PWM signal, processed by the controller by calculating a mean value of two measured values and transmitted by the USB interface with a sampling time of $0.5 \mathrm{~ms}$. The lower diagram shows the current measurement recorded with the oscilloscope, where the charging and discharging of the coil becomes visible. As derived in Section 3.1, the controller parameters

Table 1. System properties.

\begin{tabular}{lc}
\hline Description & Value \\
\hline \hline Direct current (DC) link voltage & $30 \mathrm{~V}$ \\
Coil turns $N$ & $2 \times 70$ \\
Inductance $L$ & $389 \mu \mathrm{H}$ \\
Resistance $R$ & $1.064 \Omega$ \\
Magnet remanence $B_{R}$ & $0.87 \mathrm{~T}$ \\
PWM $f_{P W M}$ & $10 \mathrm{kHz}$ \\
Sample time of USB data acquisition & $0.5 \mathrm{~ms}$ \\
Maximum current & $5 \mathrm{~A}$ \\
Minimum current & $-2.5 \mathrm{~A}$ \\
Moving mass & $5.3 \mathrm{~kg}$ \\
Reference air gap & $1.1 \mathrm{~mm}$ \\
\hline
\end{tabular}

Table 2. Controller properties.

\begin{tabular}{lc}
\hline Description & Value \\
\hline \hline Sample time of current control loop & $50 \mu \mathrm{s}$ \\
Current controller gain $K_{P, e l}$ & $1.0 \Omega$ \\
Current controller time constant $T_{N, e l}$ & $1.5 \mathrm{~ms}$ \\
Sample time of air gap control loop & $1 \mathrm{~ms}$ \\
Air gap controller gain $K_{P \mid D}$ & $-11,530 \mathrm{~A} / \mathrm{m}$ \\
Air gap controller reset time $T_{N}$ & $90 \mathrm{~ms}$ \\
Air gap controller derivative time $T_{V}$ & $10 \mathrm{~ms}$ \\
Zero current controller gain $K_{l}$ & $0.5 \mathrm{~mm} / \mathrm{As}$ \\
Spring constant $C$ & $3.5 \mathrm{k} \delta, \mathrm{Mag}$ \\
Damping ratio $\zeta$ & -0.65 \\
Undamped eigenfrequency $f_{0}$ & $29.3 \mathrm{~Hz}$ \\
Damped eigenfrequency $f_{d}$ & $22.1 \mathrm{~Hz}$ \\
\hline
\end{tabular}


follow from the electrical, differential equation and its lumped parameters. With the calculated current controller parameters, a very fast response is achieved, leading to distinct overshoots of the measured current signal. To stay within the operating range of the $\mathrm{H}$-bridge, such overshoots must be avoided. Therefore, the current controller gain $K_{P, e l}$ is slightly reduced and manually determined with respect to the calculation from $1.945 \Omega$ to $1.0 \Omega$. The same applies to the current controller time constant $T_{N, e l}$, which is calculated to $366 \mu \mathrm{s}$ and manually determined to $1.5 \mathrm{~ms}$. The step response indicates a balanced behaviour between response time and overshooting. The current settles after about $8 \mathrm{~ms}$. A set point current rectangle signal between $0 \mathrm{~A}$ and $2 \mathrm{~A}$ with a frequency of $10 \mathrm{~Hz}$ is displayed in Figure 8. The result also indicates an adequate following behaviour of the current controller.
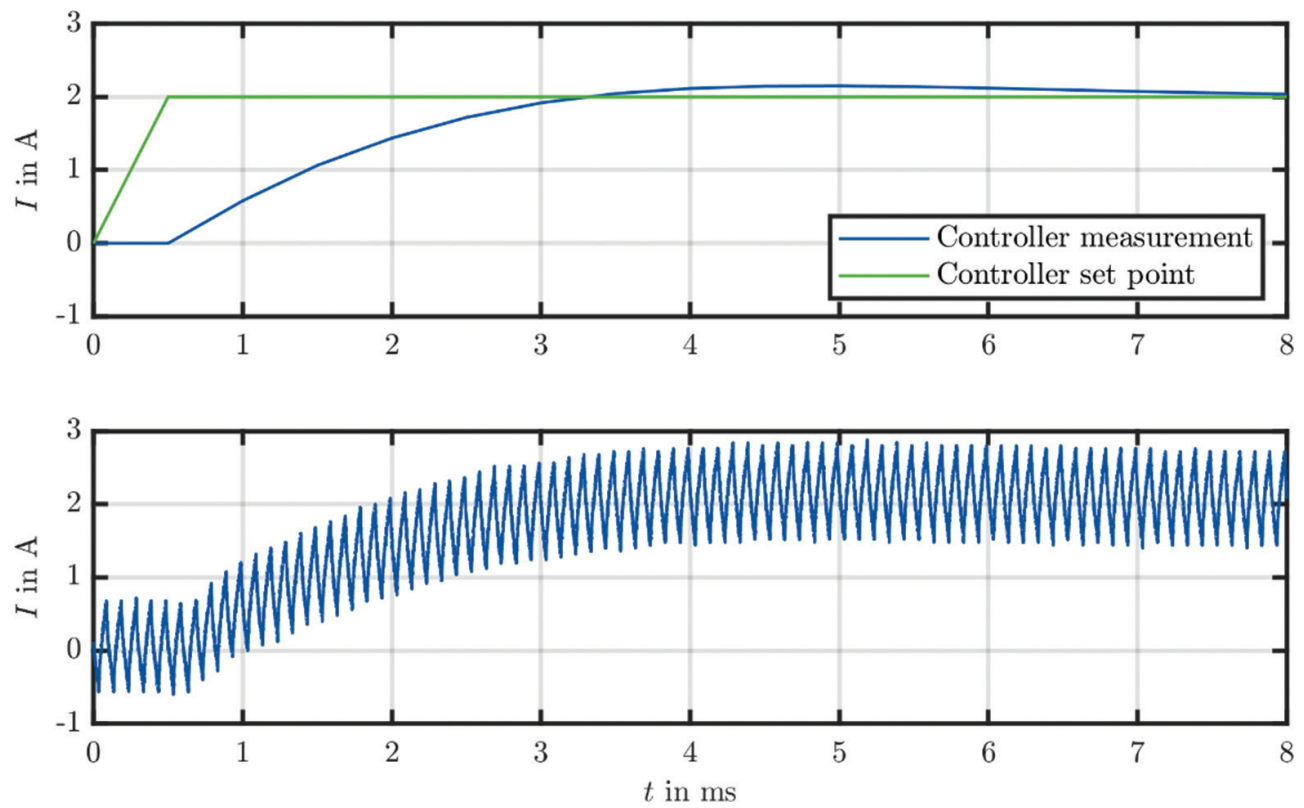

Fig. 7. The ramp of an increasing current from $0 \mathrm{~A}$ to $2 \mathrm{~A}$ (controller measurement/setpoint on top and oscilloscope measurement at bottom)

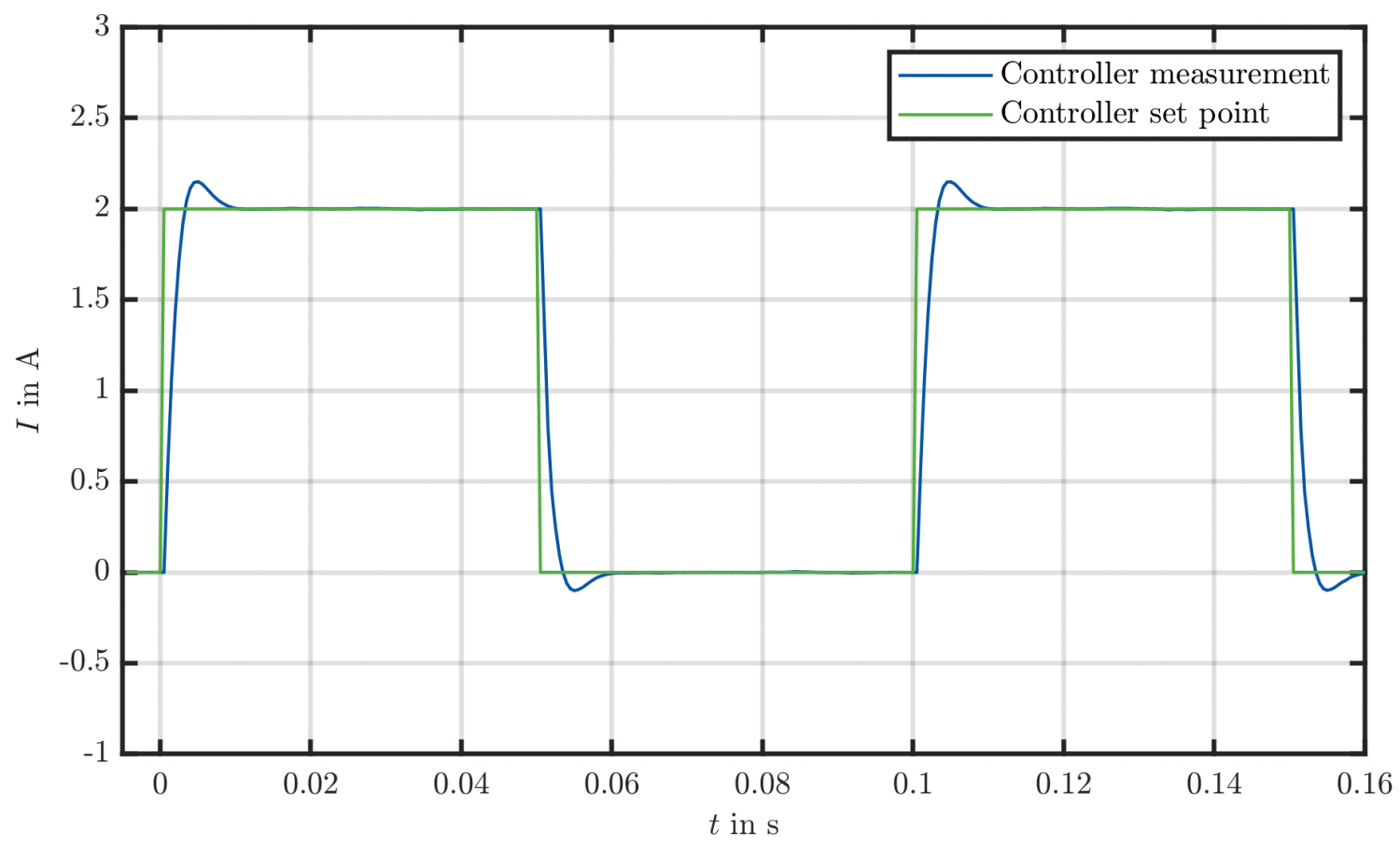

Fig. 8. Set point rectangle showing the following behaviour of the controlled current. 
Before the air gap controller is examined in detail, it has to be mentioned that when the air gap becomes smaller than the reference air gap, negative currents have to be applied to the coils to weaken the magnetic field of the PM. As the force of the magnets increases inversely proportional to the square of the air gap, the field weakening has to be applied with a small-time constant. Apart from that, the absolute value has to be limited before the knee point of the PM is reached, because otherwise, the magnets will be irreversible demagnetized (Rickwartz et al., 2020). The currents, which can be applied to strengthen or weaken the magnetic field, have to be limited to prevent the irreversible demagnetisation of the PM and stay within the permitted range of power electronics used. This results in unsymmetrical current limits given in Table 1.

To investigate the stability of the air gap controller, a set point rectangle between $1.1 \mathrm{~mm}$ and $1.0 \mathrm{~mm}$ is applied (Figure 9). As determined with the help of the zero-current controller in Figure 5, the current is close to zero at the reference air gap of $1.1 \mathrm{~mm}$ and decreases for a smaller air gap reference of $1.0 \mathrm{~mm}$. The underdamped system behaviour of a damped harmonic oscillator as derived in Section 3.2 can be observed well. The oscillation decays until $0.6 \mathrm{~s}$ and reaches a steady state. In contrast to the reference air gap of $1.1 \mathrm{~mm}$, the measured value at an air gap of $1.0 \mathrm{~mm}$ decays with oscillation too, but keeps oscillating around the set point value. This illustrates that the controller parameters are only valid for a certain operating point and the system becomes less stable when not operated tightly around the operating point. To investigate the deduced stiffness of the system, different virtual spring constants are studied. In Figure 10, the different spring constants $c$ are given as a function of $k_{\delta, M a g}$ with a constant factor $K_{c}\left(c=K_{c} \cdot k_{\delta, M a g}\right)$. As explained in Section 3.2, the stability limit is $c=2 \cdot k_{\delta, M a g}$, which indicates a stiffer system response with an increasing factor $K_{c}$. With $K_{c}=2.5$, the system is stable, but the air gap signal keeps oscillating around the set point. With an increasing factor $K_{c}$, the amplitude of the air gap oscillation decreases, while eigenfrequency and damping increase.

The behaviour can be explained by the pole-zero plot in Figure 11. The plot shows the poles and zeros of the closed air gap control loop as derived in Eq. (40) for values of $K_{c}$ from 2.5 to 4.5. It has to be noticed that this transfer function is an approximation as it assumes that the transfer behaviour of the inner current control loop is always one for all relevant operational points. For $K_{c}=2.5$ to $K_{c}=3.5$, the transfer function has one real pole, two complex conjugated poles and two zeros on the real axis, all in the left half of the s-plane. For $K_{c}=4.0$, the complex conjugated poles become poles on the real axis. As $K_{c}$ increases further, these poles approach the zeros, which increasingly reduces the influence on the transfer behaviour, while the real value of the single pole decreases for increasing $K_{c}$ and lies deeper in the left half of the s-plane, leading to a more stable system behaviour.
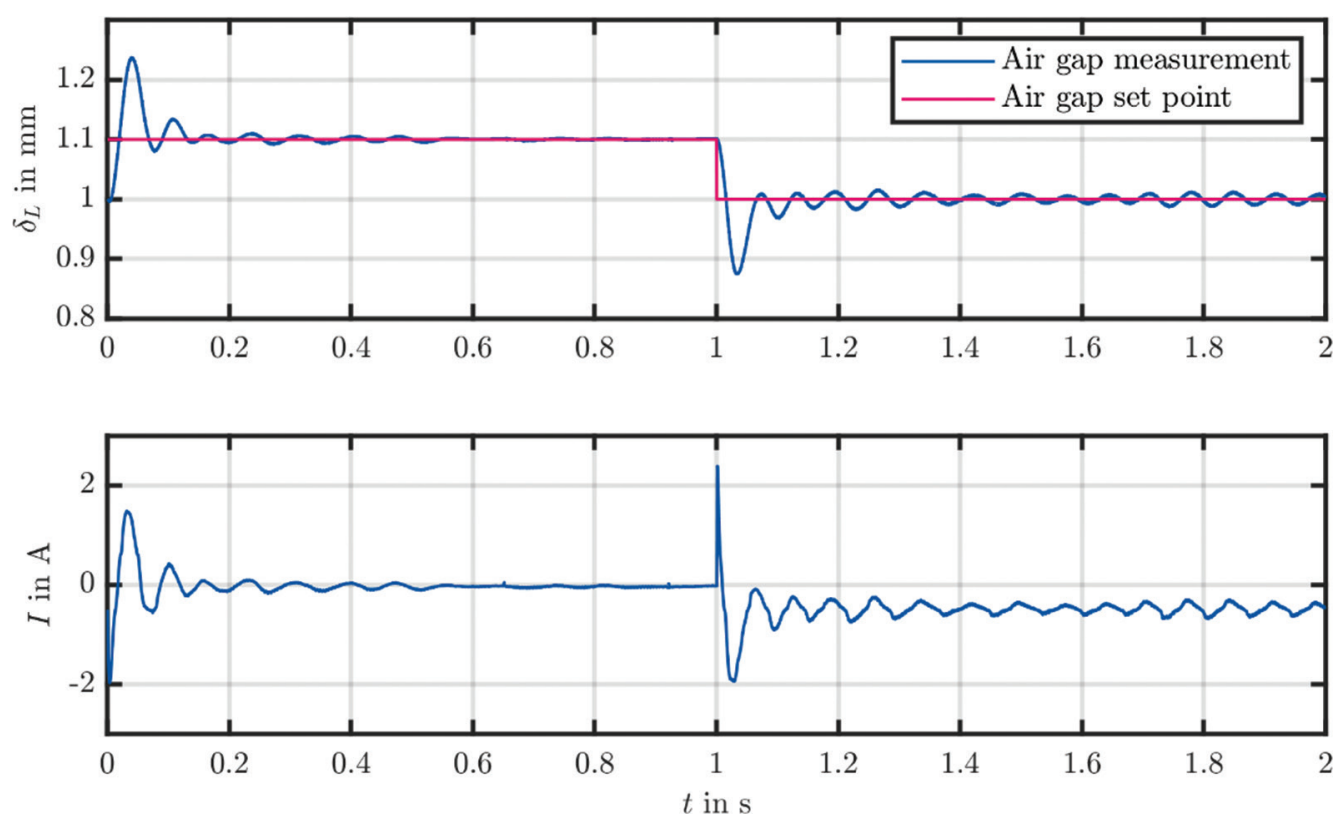

Fig. 9. Air gap and current measurement for a set point rectangle between $1.1 \mathrm{~mm}$ and $1.0 \mathrm{~mm}$. 

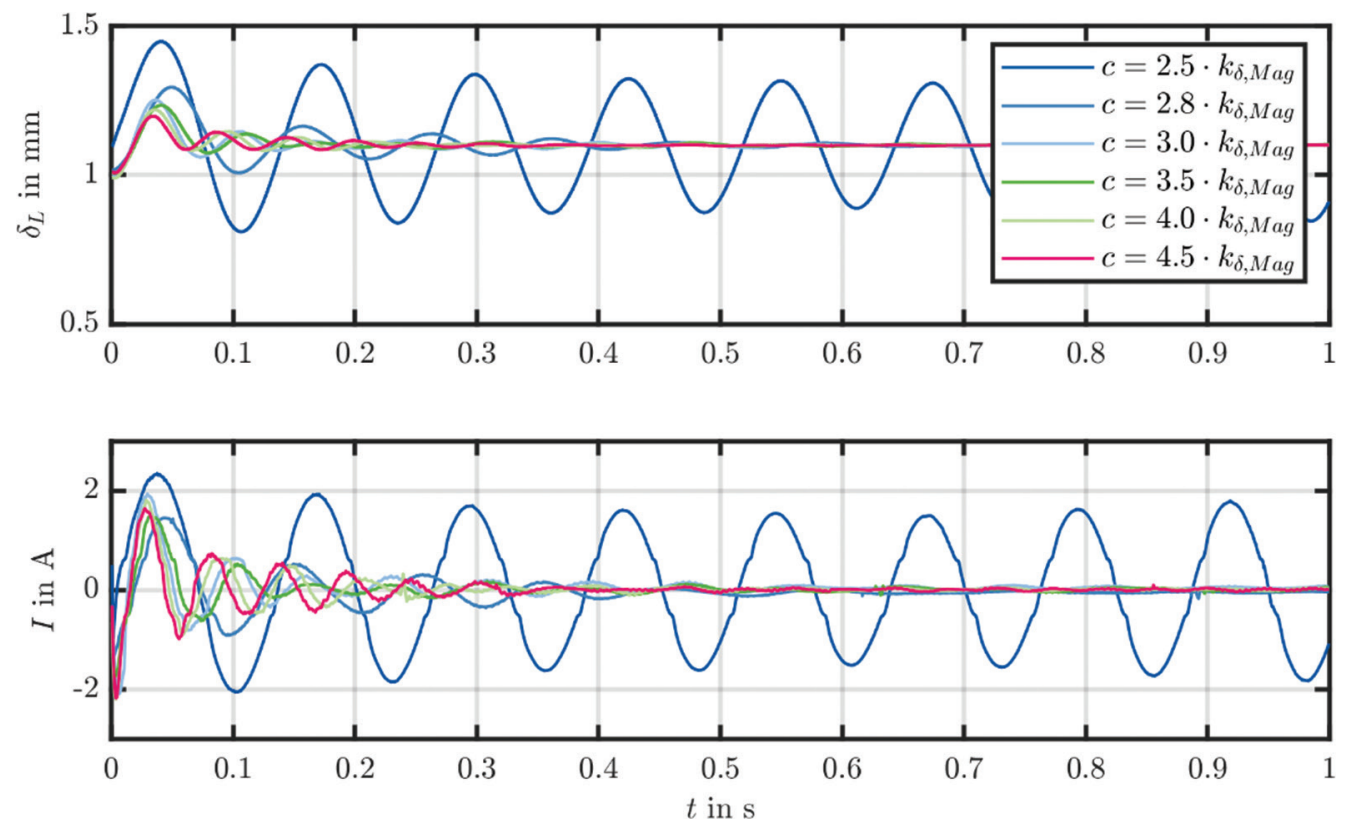

Fig. 10. Air gap and current for different spring constants $C$.

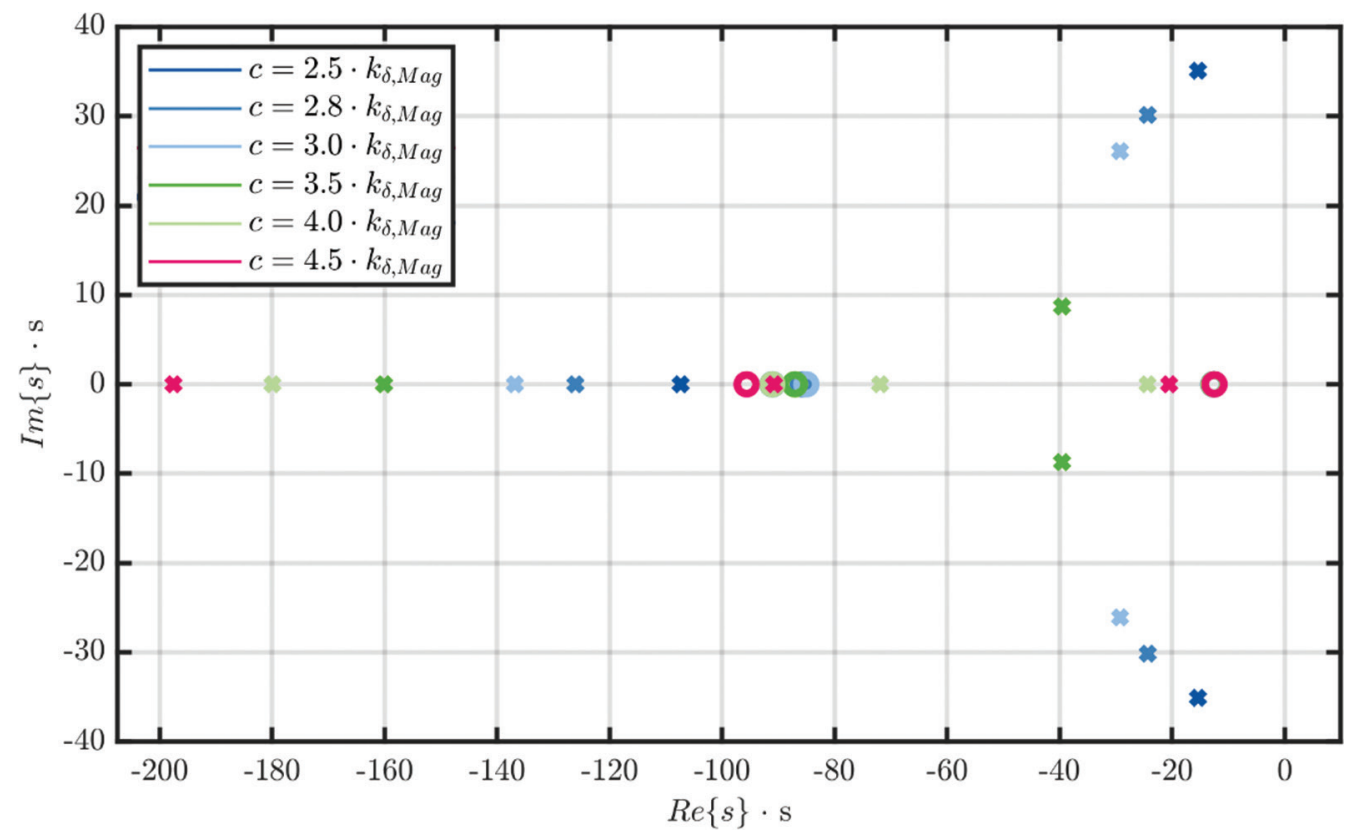

Fig. 11. Pole-zero plot of the closed-loop transfer function in Eq. (40) for different spring constants $c$.

To verify the damped eigenfrequency value, a Fast Fourier Transformation is performed on a step response with $K_{c}=3.5$ as depicted in Figure 12.

The measured air gap in Figure 12 shows the transient response to a step change of the air gap set point from $1.0 \mathrm{~mm}$ to $1.1 \mathrm{~mm}$. In the diagram of air gap vs. frequency, the resulting frequency spectrum indicates the eigenfrequency at about $14 \mathrm{~Hz}$. This is lower than the calculated damped eigenfrequency of $22.1 \mathrm{~Hz}$. The eigenfrequency decreases with higher damping, which is presumably the reason, why the measured eigenfrequency is lower. As seen in Figure 3, the hybrid actuator, which is modelled as a damped harmonic oscillator is mounted on 
a test bench. Said test bench consists of two copper plates, holding the hybrid actuator and allowing a movement in the vertical direction. On the copper plates, a weight is placed, which provides for the weight force. The effective weight force, acting on the hybrid actuator, results from the lever. The test bench introduces additional damping caused by the setup, which is neglected in the controller design.

If the controller parameters given in Table 2 are applied, Figure 13 shows the air gap and current signal of the actuator for different air gap set points between $0.8 \mathrm{~mm}$ and $1.3 \mathrm{~mm}$. The air gap set point change is ramped with a slope of $10 \mathrm{~mm} / \mathrm{s}$. It can be observed that for the reference value at $1.1 \mathrm{~mm}$, but also for all other examined operational points, the system is stable with a fast response.
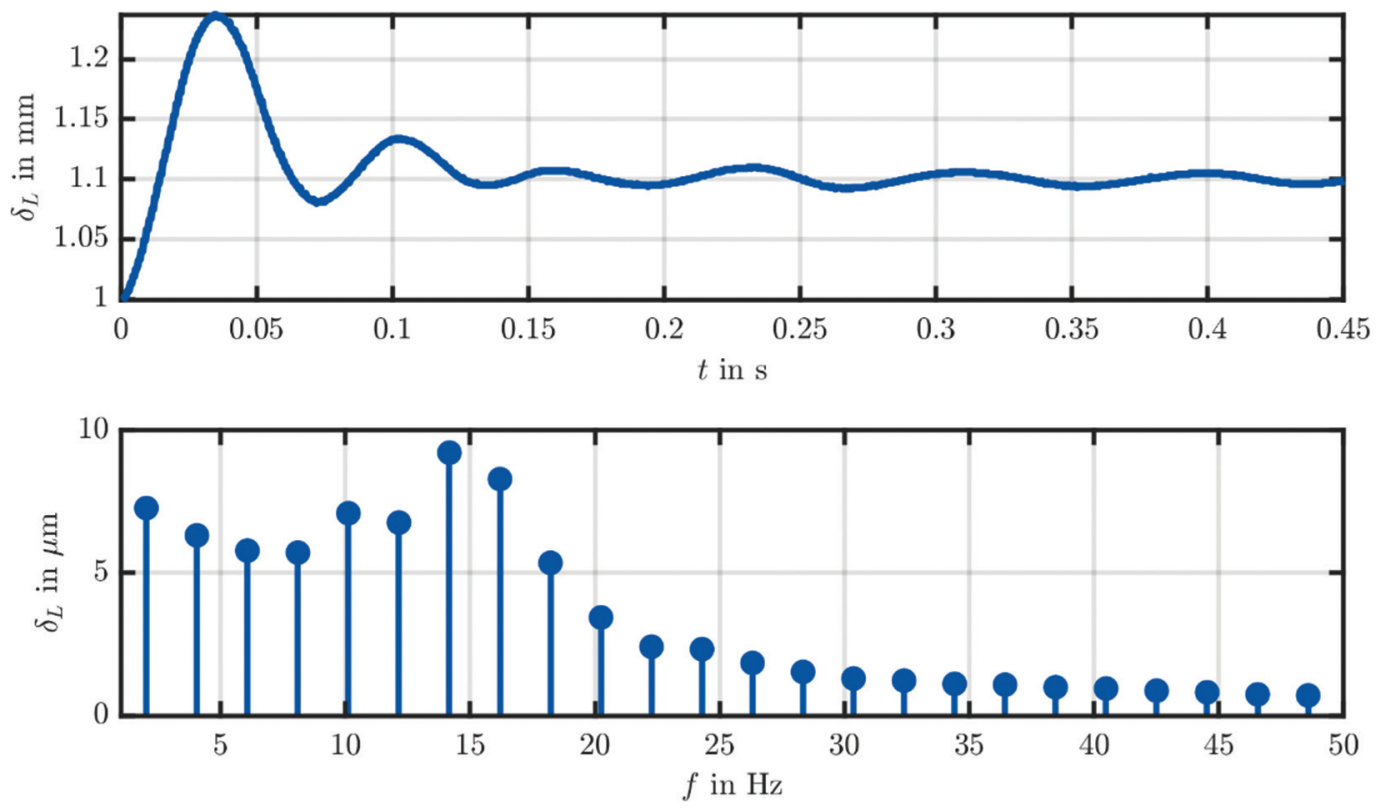

Fig. 12. Transient response and spectrum analysis showing the eigenfrequency at $14 \mathrm{~Hz}$ with $K_{\mathrm{c}}=3.5$.
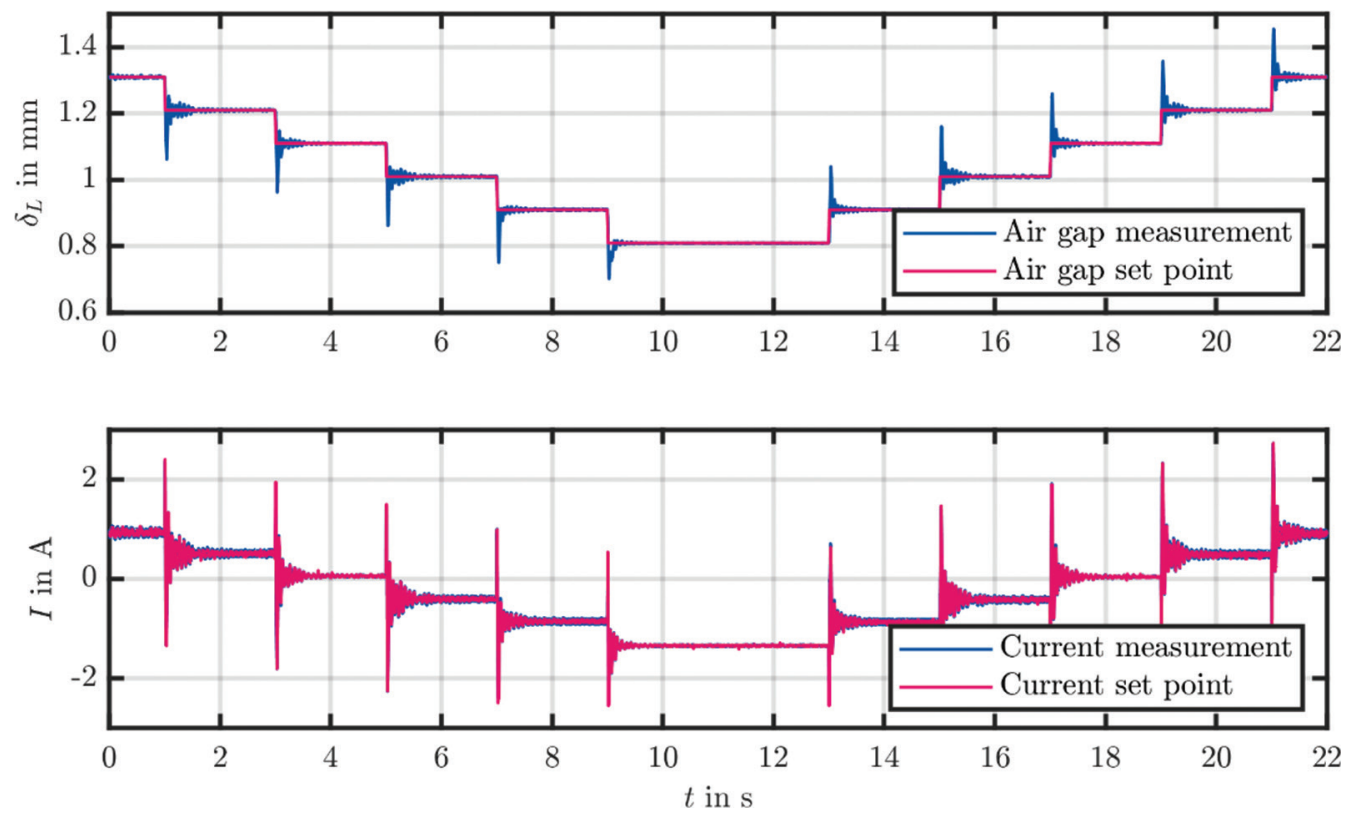

Fig. 13. Different set point air gaps showing the responses in the air gap and current measurement. 


\section{Conclusions}

In this paper, an analytical approach to determine controller parameters for the closed-loop air gap control of a hybrid actuator for magnetic levitation applications is presented. The hybrid actuator consists of a combination of PM and electromagnets and can be designed so that the PM hold a certain static load, while the electromagnets are currentcontrolled and fed by dynamic currents resulting in dynamic forces stabilising the inherently unstable system in this operational point. The basic theory of a magnetic circuit, applied to the plain hybrid actuator geometry in this paper, is presented in Section 2, resulting in an equation describing the total force resulting from the combination of PM and electromagnets. It is shown that the total magnetic force is, besides various constants, dependent on the current and the air gap. Therefore, the derivation of the controller in Section 3 starts with the balance of forces and divides it into a static part, where the forces compensate for a certain air gap and a dynamic part, where the electromagnets are used to stabilise the system around the design point of the actuator system. Section 3.2 focusses on the dynamic balance of forces and presents a controller design, which changes the unstable, uncontrolled system behaviour of the hybrid actuator into the stable and controllable system behaviour of a damped harmonic oscillator. It is shown that the controller parameters of the outer air gap control loop can be used to set the spring constant $c$ of the damped harmonic oscillator, which then determines the stability behaviour of the controlled hybrid actuator.

In Section 4, test bench setup and control and measurement electronics are introduced. The hybrid actuator is mounted on a test bench, which allows a movement of the actuator in the vertical direction, while it is carried by two copper plates. The test bench closes the magnetic circuit of the hybrid actuator with an iron yoke. Apart from that, a variable mass is placed on the test bench to increase the weight force, acting on the hybrid actuator. The control algorithm is executed on an MCU and a self-manufactured shield board is stacked on the MCU, used to provide different supply voltages and to carry the ADC unit to convert analogue air gap and current signal to digital signals processed by the MCU. For current control, a full-bridge inverter is used and the air gap is measured by an eddy current sensor. The test bench and measurement hardware are then used to carry out various measurements. First, the reference air gap, meaning the nominal air gap, where no DC is necessary to levitate the hybrid actuator, is determined. To evaluate the exact reference air gap, a zero-current control loop is implemented additionally to the air gap controller to determine all relevant parameters for calculating controller parameters in the reference operational point. Afterwards, the current controller is evaluated. As the behaviour of this control loop is the basis for a fast, stable and reliable air gap control, the aim is to adjust the current controller so that fast response and moderate overshoots are in good proportion to each other.

The air gap controller is evaluated for different controller parameters, which are determined by defining a spring constant according to theoretical constraints. Limits for a stable operation of the hybrid actuator have been derived. These limits can be verified by measurements and are discussed with the help of a pole-zero plot. The damped eigenfrequency of the system is determined by the Fast Fourier Transformation of the system's response on an air gap step. In a final experiment, the system behaviour around the reference air gap is analysed by systematically varying the air gap set points between $0.8 \mathrm{~mm}$ and $1.3 \mathrm{~mm}$ with a set point slope of $10 \mathrm{~mm} / \mathrm{s}$. The system shows a stable behaviour for all operational points, although it is designed for a reference value of $1.1 \mathrm{~mm}$.

\section{References}

Bierhoff, M. H. and Fuchs, F. W. (2009). Active Damping for Three-Phase PWM Rectifiers with High-Order Line-Side Filters. IEEE Transactions on Industrial Electronics, 56(2), pp. 371-379. https://doi. org/10.1109/TIE.2008.2007950.

Bohn, G. and Steinmetz, G. (1984). The Electromagnetic Levitation and Guidance Technology of the 'Transrapid' Test Facility Emsland. IEEE Transactions on Magnetics, 20(5), pp. 1666-1671. https://doi.org/10.1109/TMAG.1984.1063246.

Cervera, A., Ezra, O., Kuperman, A. and Peretz, M. M. (2019). Modeling and Control of Magnetic Actuation
Systems Based on Sensorless Displacement Information. IEEE Transactions on Industrial Electronics, 66(6), pp. 4849-4859. https://doi. org/10.1109/TIE.2018.2847652.

Cho, H.-W., Yu, J.-S., Jang, S.-M., Kim, C.-H., Lee, J.-M. and Han, H.-S. (2012). Equivalent Magnetic Circuit Based Levitation Force Computation of Controlled Permanent Magnet Levitation System. IEEE Transactions on Magnetics 48(11), pp. 4038-4041. https://doi.org/10.1109/TMAG.2012.2198800.

He, J. L., Rote, D. M. and Coffey, H. T. (1994). Study of Japanese Electrodynamic-Suspension Maglev 
Systems. Argonne National Lab., IL (United States). Energy Systems Division ANL/ESD-20. https://doi.org/10.2172/10150166.

Henzel, M. and Mazurek, P. (2011). The analysis of the control system of the active magnetic bearing. In: Z. T. Bronislaw, ed., Electrodynamic and Mechatronic Systems (SCE III). Opole, Poland, 6-8 October 2011, In: 2011 IEEE 3rd International Students Conference on Electrodynamics and Mechatronics (SCE III), Opole, Poland, 10 June 2011 - 10 August 2011, IEEE: Piscataway, NJ, pp. 53-58.

Kim, C.-H., Cho, H.-W., Lee, J.-M., Han, H.-S., Kim, B.-S., Kim, D.-S. (2010). Zero-power control of magnetic levitation vehicles with permanent magnets. In: 2010 International Conference on Control Automation and Systems (ICCAS 2010), Gyeonggido, 27 October 2010 - 30 October 2010, IEEE, pp. 732-735.

Kim, K.-J., Han, H.-S., Kim, C.-H. and Yang, S.-J. (2013). Dynamic Analysis of a Maglev Conveyor Using an EM-PM Hybrid Magnet. Journal of Electrical Engineering and Technology, 8(6), pp. 1571-1578.

Kim, Y. H., Kim, K. M. and Lee, J. (2001). Zero Power Control with Load Observer in Controlled-PM Levitation. IEEE Transactions on Magnetics, 37(4), 2851-2854. https://doi.org/10.1109/20.951326.

Lin, C. E. and Lin, K. G. (2000). Implementation and control of the magnetic linear actuation system. In: Proceedings of the 17th IEEE Instrumentation and Measurement Technology Conference, IMTC 2000, Baltimore, MD, USA, 1-4 May 2000, IEEE, pp. 1384-1387.
Papadopoulos, K. (2015). PID Controller Tuning Using the Magnitude Optimum Criterion. Cham: Springer International Publishing.

Rickwartz, J. P., Kolb, J. and Hameyer, K. (2020). Control, simulation and validation of a hybrid actuator for a Maglev train model on a scale of 1:20. In: 2020 21st International Conference on Research and Education in Mechatronics (REM), Cracow, Poland, 12 September 2020 - 12 November 2020, IEEE, pp. 1-6.

Shin, H.-J., Choi, J.-Y., Jung, K.-H., Lee, J.-M. and Kim, C.-H. (2016). Influence of Lateral-Impact Force on Electropermanent Magnet Suspension Conveyor with Inherent Guidance Force. IEEE Transactions on Magnetics, 52(7), pp. 1-4. https:// doi.org/10.1109/TMAG.2016.2514298.

Zhang, C., Nguyen, T. D., Tseng, K. J. and Zhang, S. (2010). Stiffness analysis and levitation force control of the active magnetic bearing for a partially-self-bearing flywheel system. In: 2010 IEEE International Conference on Sustainable Energy Technologies (ICSET 2010), Kandy, Sri Lanka, 6-9 December 2010, Kandy, Sri Lanka, 12 June 2010 - 12 September 2010, Piscataway, NJ: IEEE, pp. 1-6.

Zhao, C., Sun, F., Jin, J., Tang, H. J. and Xu, F., Li, Q. and Oka, K. (2020). Analysis of Quasi-Zero Power Characteristic for a Permanent Magnetic Levitation System with a Variable Flux Path Control Mechanism. IEEE/ASME Transactions on Mechatronics, 1. https://doi.org/10.1109/ TMECH.2020.3026086. 